\title{
Methodological concern. (Mini-commentary on BJOG-20-0063.R1)
}

\author{
Steven Lane ${ }^{1}$ \\ ${ }^{1}$ University of Liverpool
}

May 6, 2020

Mini-commentary on BJOG-20-0063.R1: Association of maternal attention deficit hyperactivity disorder and preterm birth: a cohort study

\section{Methodological concern}

Steven Lane

University of Liverpool

Institute of Translational Medicine

Liverpool, UK

Email: Slane@liverpool.ac.uk

A note of caution is advised on the definition of a woman considered to have ADHD at some time-point before, during or post pregnancy. In the reported study women were considered to have had an episode of ADHD if they were dispensed a prescription for an ADHD medication (Hesselman et al. BJOG 2020 $\mathrm{xxxx}$ ). This will not be as accurate as a clinical diagnosis of the condition. Dispensing medication depends on the women presenting the prescription at a pharmacy, which is not always the case when mental health issues are prevalent. There is, therefore, a strong possibility that the estimates of women with and without ADHD are inaccurate and that both groups contain a mixture of women both with and without ADHD. As it is likely that some prescriptions were not presented then, statistically, it is likely that the number of women with ADHD is underestimated. It should also be noted that, in this cohort of women, medication is only prescribed if other supportive measures have failed, again suggesting an under estimation of true ADHD cases, and that the prescribed medications could be prescribed for other mental health conditions, this time possibly over estimating ADHD cases. In summary, this is an interesting study and the authors have highlighted the issues relating to the accuracy of the definition of an ADHD case in the discussion section, readers should consider these limitations when interpreting the results of the study.

No disclosures: A completed disclosure of interest form is available to view online as supporting information. 